\title{
Modelling molecular gas suspension diffusion and saturation processes in liquid media
}

\author{
R. Groll \\ Center of Applied Space Technology and Microgravity, \\ University of Bremen, Germany
}

\begin{abstract}
A model describing the suspension diffusion process of gas molecules in liquid media is presented in this paper. This process is not yet solved by a satisfactory model for micro-scale applications at this time. The new approach allows the simulation of diffusion processes in continuous media considering the molecular mass flux in a suspension/carrier phase mixture. Modelling the diffusion of gas suspensions in liquid media the saturation mass ratio is reached near the liquid/gas surface very quickly. The increase of gas concentration in the liquid domain depends on the elapsed time and the physical properties of gas and liquid media. The molecular gas velocity is described by a Maxwell probability density function. Modelling the gas species diffusion the molecular convection is considered. Modelling the mass flux of the molecular gas suspension characteristic time scales are developed describing the completion level of the saturation progress based on non-dimensional formulations of the molecular convection equation. The present model is implemented in a CFD code and validated by a family of parametric simulation results depending on the saturation mass ratio of the suspended gas phase. This simulation result array shows the dependency of saturation time and saturation mass ratio of the suspended gas molecules. Based on this relation macroscopic diffusion processes in micromixers and microchannels are described with this model and without an extra solution of molecule trajectories or spectral fields of molecule velocity.

Keywords: two-phase flow, molecular diffusion, gas/liquid, dispersion, saturation.
\end{abstract}




\section{Introduction}

The phase interaction of multiphase systems is a topic which is investigated for the last century $[6,11,12,15]$ without giving clear answers modeling the diffusion process of a suspended disperse phase in a continuous carrier phase $[19,20]$. Modelling the transport of suspended gas molecules in a liquid carrier phase can not be described by discrete models $[3,5,10,16]$. The motion of suspended molecules follows diffusion characteristics $[1,4,7,13,18]$ as described in temperature or diffusive combustion processes. The major boundary condition modelling the gas suspension process is the start concentration of the suspension at the phase interface. The stability of numerical simulation depends on the sensitivity of such a boundary condition [9, 17]. Modelling the mass transport at this boundary condition defines the global mass balance and is are significant parameter for the quantitative results [2]. Finally basic approximations of molecular motions are necessary to give better values to be considered for construction and development of future advanced mixing devices.

\section{Continuum mechanical approach}

In Fig. 1 the mass transport procedure at the phase interface is shown. During this suspension process the liquid phase of the species $(k=F)$ absorbs gas molecules of the species $(k=G)$. The partial densities of both phases $\rho^{k}$ are defined by the product of the molecule mass of liquid phase molecules or gas phase molecules $m^{k}$ and the number density $n^{k}$, which means the molecule number per volume in a given mixture volume $V$.

$$
\begin{aligned}
& \rho^{F}=m^{F} \frac{N_{V}^{F}}{V}=m^{F} n^{F} \\
& \rho^{G}=m^{G} \frac{N_{V}^{G}}{V}=m^{G} n^{G}
\end{aligned}
$$

Supposing that suspended gas molecules replace no fluid molecule the efficient mixture density $\rho^{*}$ is defined by the sum of the partial densities of gas and liquid:

$$
\rho^{*}=\rho^{F}+\rho^{G}
$$

The concentration $c^{k}$ of the species $k$ is defined by the ratio of partial density and mixture density:

$$
c^{k}=\frac{\rho^{k}}{\rho^{*}}
$$

Based on the concentration definition of fluid and gas phase media the partial density $\rho^{k}$ is defined on concentration $c^{k}$ and the efficient micture density $\rho^{*}$ :

$$
\begin{aligned}
c^{F}+c^{G} & =1 \\
\rho^{*} c^{k} & =\rho^{k}
\end{aligned}
$$


Because of the continuity of both species the corresponding equations for the partial densities are defined depending on the partial velocities of of the liquid and gaseous phase:

$$
\begin{aligned}
& \frac{\partial}{\partial t} \rho^{F}+\frac{\partial}{\partial x_{j}}\left(\rho^{F} u_{j}^{F}\right)=0 \\
& \frac{\partial}{\partial t} \rho^{G}+\frac{\partial}{\partial x_{j}}\left(\rho^{G} u_{j}^{G}\right)=0
\end{aligned}
$$

Summarizing these equations the global mass balance results:

$$
\begin{aligned}
0 & =\frac{\partial}{\partial t}(\underbrace{\rho^{F}+\rho^{G}}_{\rho^{*}})+\frac{\partial}{\partial x_{j}}\left(\rho^{F} u_{j}^{F}+\rho^{G} u_{j}^{G}\right) \\
& =\frac{\partial}{\partial t} \rho^{*}+\frac{\partial}{\partial x_{j}}(\rho^{*} \underbrace{\frac{\rho^{F} u_{j}^{F}+\rho^{G} u_{j}^{G}}{\rho^{F}+\rho^{G}}}_{=: U_{j}^{*}}) \\
& =\frac{\partial}{\partial t} \rho^{*}+\frac{\partial}{\partial x_{j}}\left(\rho^{*} U_{j}^{*}\right)
\end{aligned}
$$

This equation is the continuity equation of the liquid/gas mixture of the species $G$ and $F$. The global mass flow results from the effective mixture density $\rho^{*}$ and the effective mixture velocity $U^{*}$.

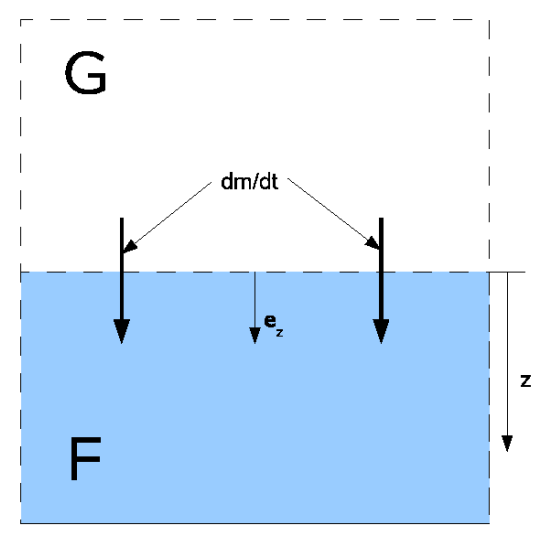

Figure 1: Distributed phases: $G$ (gas) is suspended in phase $F$ (fluid) with the mass flow $d m / d t$ at the phases interphase. 


\subsection{Concentration of the suspension}

To get a transport equation for the concentration, the concentration depending density $\rho^{k}$ (eq. (6)) is substituted in the continuity equation (eq.(7)):

$$
\frac{\partial}{\partial t}\left(\rho^{*} c^{F}\right)+\frac{\partial}{\partial x_{j}}\left(\rho^{*} c^{F} u_{j}^{F}\right)=0,
$$

For this following transport equation with a mixture velocity depending convective term results:

$$
\begin{gathered}
\frac{\partial}{\partial t}\left(\rho^{*} c^{F}\right)+\frac{\partial}{\partial x_{j}}\left(\rho^{*} c^{F} U_{j}^{*}\right) \\
=\frac{\partial}{\partial x_{j}}\left[\rho^{*} c^{F}\left(U_{j}^{*}-u_{j}^{F}\right)\right] \\
=\frac{\partial}{\partial x_{j}}\left[c^{F}(\underbrace{\rho^{*} U_{j}^{*}}_{=\rho^{F} u_{j}^{F}+\rho^{G} u_{j}^{G}}-\rho^{*} u_{j}^{F})\right] \\
=\frac{\partial}{\partial x_{j}}[c^{F}\left(\rho^{G} u_{j}^{G}\right)+\underbrace{\left(c^{F}-1\right)}_{=-c^{G}}\left(\rho^{F} u_{j}^{F}\right)]
\end{gathered}
$$

Based on a substitution with one of the both concentration terms (eq. (5)) the transport equation of the species concentration results:

$$
\begin{aligned}
& \frac{\partial}{\partial t}\left(\rho^{*} c^{F}\right)+\frac{\partial}{\partial x_{j}}\left(\rho^{*} c^{F} U_{j}^{*}\right) \\
= & \frac{\partial}{\partial x_{j}}\left[c^{F}\left(\rho^{G} u_{j}^{G}\right)-c^{G}\left(\rho^{F} u_{j}^{F}\right)\right]
\end{aligned}
$$

Here a transport equation depending on the mixture density $\rho^{*}$ and the mixture velocity $U^{*}$ analogous to the transport equation of other physical values of this mixture process is given.

\subsection{Modelling the diffusion approach}

Based on the consideration, that the developed diffusion term is modelable by the linear approximation, the transport follows from Fick's law:

$$
\frac{\partial}{\partial t}\left(\rho^{*} c^{k}\right)+\frac{\partial}{\partial x_{j}}\left(\rho^{*} c^{k} U_{j}^{*}\right)=\frac{\partial}{\partial x_{j}}\left(\rho^{*} D \frac{\partial c^{k}}{\partial x_{j}}\right)
$$


and following equivalence from eq. (12):

$$
\rho^{*} D \frac{\partial c^{F}}{\partial x_{j}}=c^{F}\left(\rho^{G} u_{j}^{G}\right)-c^{G}\left(\rho^{F} u_{j}^{F}\right)+A_{j}
$$

with a constant vector $\vec{A}$.

It is considered with the hypothesis $\left|\vec{u}^{F}\right|=0$ that the motion of the liquid medium is negligible during the diffusion process of the gaseous molecules. By the upper result (eq. (14)) following relation of the concentration gradient results:

$$
\rho^{*} D \frac{\partial c^{F}}{\partial x_{j}}=c^{F}\left(\rho^{G} u_{j}^{G}\right)+A_{j}
$$

At the wall for all species $k$ it is given:

$$
\left.\frac{\partial c^{k}}{\partial x_{j}}\right|_{W}=0 \quad,\left.\quad u_{j}^{k}\right|_{W}=0
$$

From eq. (15) the constant vector $\vec{A}=\overrightarrow{0}$ results and considering eq. (6) the mixture velocity is given:

$$
\begin{aligned}
u_{j}^{G} & =\frac{\rho^{*}}{c^{F} \rho^{G}} D \frac{\partial c^{F}}{\partial x_{j}} \\
& =\frac{D}{c^{F} c^{G}} \frac{\partial c^{F}}{\partial x_{j}}
\end{aligned}
$$

By this definition the global relation between diffusion rate and concentration gradient is shown.

\subsection{Mass flow rate at the phase interface}

Based on the assumption that the suspended gas phase reaches the saturation concentration at the phase interface (PI)

$$
\left.c^{G}\right|_{P I}=c_{\max }^{G}
$$

the interfacial mass flow is given in the following way:

$$
\begin{aligned}
\left.u_{j}^{G}\right|_{P I} & =\left.\frac{D}{\left(1-c_{\max }^{G}\right) c_{\max }^{G}} \frac{\partial c^{F}}{\partial x_{j}}\right|_{P I} \\
& =\left.\frac{D}{\left(c_{\max }^{G}-1\right) c_{\max }^{G}} \frac{\partial c^{G}}{\partial x_{j}}\right|_{P I} \\
\left.\dot{m}\right|_{P I} & =\left(\rho^{*} c^{G} \vec{u}^{G} \cdot \vec{e}_{z}\right)_{P I}=\left.\frac{\rho^{*} D}{c_{\max }^{G}-1} \frac{\partial c^{G}}{\partial z}\right|_{P I}
\end{aligned}
$$

Because of the dependence of the mixture density $\rho^{*}$ from the partial suspension density $\rho^{G}$ and therefore from the suspension concentration it is substituted by 
$\rho^{*}=\rho^{F} / c^{F}$. So the following explicit form of the interfacial mass flow results:

$$
\left.\dot{m}\right|_{P I}=-\left.\underbrace{\frac{\rho^{F} D}{\left(1-c_{\text {max }}^{G}\right)^{2}}}_{=\text {const. }} \frac{\partial c^{G}}{\partial z}\right|_{P I}
$$

With this form the interfacial mass flow is defined by the initial density of the liquid phase (without suspension) $\rho^{F}$, the saturation concentration $c_{\max }^{G}$, the binary diffusion coefficient $D$ and the local concentration gradient of the suspended species.

\section{Transport equations}

Inducing the resulting suspension velocity (eq. (17)) the continuity equation of the suspended gaseous phase is given by following equation:

$$
\frac{\partial}{\partial t} \rho^{G}+\frac{\partial}{\partial x_{j}}\left(\frac{\rho^{G} D}{c^{F} c^{G}} \frac{\partial c^{F}}{\partial x_{j}}\right)=0
$$

By eq. (6) following transport equation results independent from the suspension velocity:

$$
\begin{array}{r}
\frac{\partial}{\partial t}\left(\rho^{*} c^{G}\right)=\frac{\partial}{\partial x_{j}}\left(\frac{\rho^{*} D}{1-c^{G}} \frac{\partial c^{G}}{\partial x_{j}}\right) \\
\text { with } \quad \rho^{*}=\frac{\rho^{F}}{1-c^{G}}
\end{array}
$$

The implicit form of this equation is unsuitable for linear solvers:

$$
\begin{aligned}
\frac{\partial}{\partial t}\left(\frac{c^{G}}{1-c^{G}}\right) & =\frac{\partial}{\partial x_{j}}\left(\frac{D}{\left(1-c^{G}\right)^{2}} \frac{\partial c^{G}}{\partial x_{j}}\right) \\
& =\frac{\partial}{\partial x_{j}}\left[D \frac{\partial c^{G}}{\partial x_{j}}\left(\frac{c^{G}}{1-c^{G}}\right)\right]
\end{aligned}
$$

In analogy to the exact solution of the implicit formulation the ratio $c^{G} /\left(1-c^{G}\right)$ is the exact solution of a mathematical heat equation (eq. (24)) in a non-restricted domain:

$$
\frac{c^{G}}{1-c^{G}}=\frac{c_{\max }^{G}}{1-c_{\max }^{G}}\left[1-\operatorname{erf}\left(\frac{x}{2 \sqrt{D t}}\right)\right]
$$


After a corresponding conversion the explicit formulation of the suspension concentration $c^{G}$ results depending on spatial position and time.

$$
\begin{aligned}
\frac{1}{c^{G}}-1 & =\frac{\frac{1}{c_{\max }^{G}}-1}{1-\operatorname{erf}\left(\frac{x}{2 \sqrt{D t}}\right)} \\
c^{G} & =\left[1+\frac{\frac{1}{c_{\max }^{G}}-1}{1-\operatorname{erf}\left(\frac{x}{2 \sqrt{D t}}\right)}\right]^{-1} \\
& =\left[1+\frac{1-c_{\max }^{G}}{c_{\max }^{G}\left(1-\operatorname{erf}\left(\frac{x}{2 \sqrt{D t}}\right)\right)}\right]^{-1}
\end{aligned}
$$

This exact approach is valid for a non-restricted domain only, where the suspension diffuses.

\section{Simulation results and verification}

In a simulation the proposed mass flow inlet boundary condition (eq. (21)) is verified. The input/start requirement concentration is zero over complete domain at $t=0$. This is the initial condition for the diffusion process simulation. At the phase interface only $(x=0)$ the concentration of the suspended gas is equivalent with the saturation concentration $c_{\max }$.

Based on this initial condition two simulations are performed. Verifying the mass flow model at the phase interface the simulation results of the boundary condition with a given concentration at the interface $\left.c\right|_{x=0}=c_{\max }$ (Inlet 1) are compared with the proposed mass flow boundary condition (Inlet 2). For the comparing simulations as the exact solution $c_{\max }=0,2, L=0.1 \mathrm{~m}$ and $D=10^{-3} \frac{\mathrm{m}^{2}}{\mathrm{~s}}$ are used.
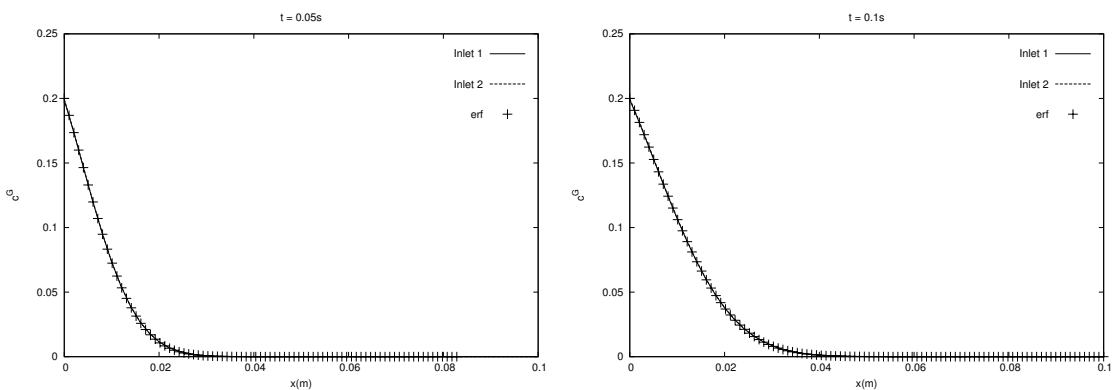

Figure 2: Computational simulations of the concentration distribution (Inlet 1, Inlet 2) are conform with the error function (erf) away from a wall. 
Now it is shown that during the time, when the concentration at the wall is negligible, the influence of the wall is also negligible and the simulation result of both inlet conditions (Fig. 2) are equivalent with the exact solution of a diffusion process in an open domain (erf, eq .26).

After reaching the wall of the higher concentrations (Fig. 3), simulation results of eq. (26) diverge from the open domain solution, because this exact solution is not defined in restricted volumes. But because of the equivalence of both simulation results with the initial and the modelled mass flow boundary condition, the mass flow model at the phase interface is verified for open and restricted domains.

\section{Saturation of a restricted domain}

Describing the time-dependent saturation eq. (23) is dedimensioned. All variable values are normalised with the constants $L, \rho^{F}$ and $D$, so that the normalized values are non-dimensional. For this transformation the equation is defined depending on:

$$
t_{+}=\frac{t D}{L^{2}} \quad, \quad x_{+}=\frac{x}{L} \quad, \quad \rho_{+}=\frac{\rho^{*}}{\rho^{F}}
$$

by multiplying eq. (23) with the ratio $L^{2} /\left(D \rho^{F}\right) . L$ is the characteristic length of the restricted domain:

$$
\begin{aligned}
\frac{\partial}{\partial\left(\frac{t D}{L^{2}}\right)}\left(\frac{\rho^{*}}{\rho^{F}} c^{G}\right) & =\frac{\partial}{\partial\left(\frac{x_{j}}{L}\right)}\left(\frac{\rho^{*}}{\rho^{F}} \frac{1}{1-c^{G}} \frac{\partial c^{G}}{\partial\left(\frac{x_{j}}{L}\right)}\right) \\
\Rightarrow \quad \frac{\partial}{\partial t_{+}}\left(\rho_{+} c^{G}\right) & =\frac{\partial}{\partial x_{+j}}\left(\frac{\rho_{+}}{1-c^{G}} \frac{\partial c^{G}}{\partial x_{+j}}\right)
\end{aligned}
$$
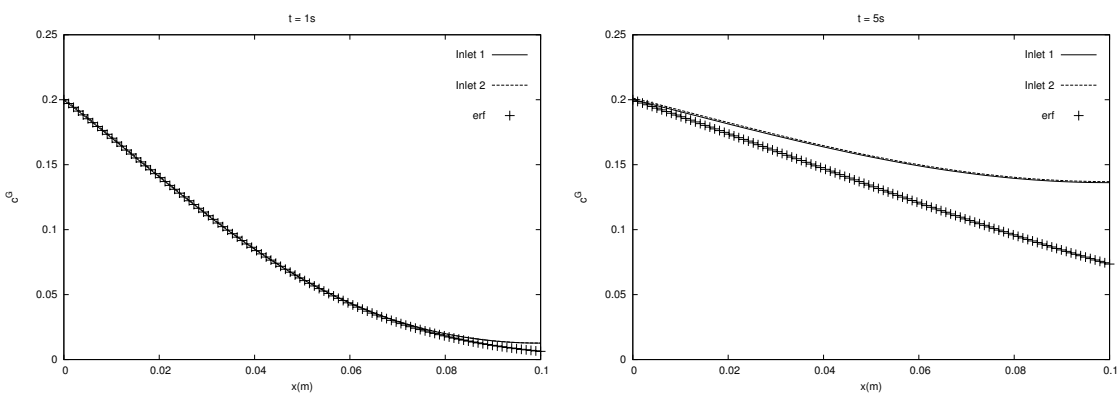

Figure 3: Computational solution (Inlet 1, Inlet 2) diverge from the exact open domain solution when the concentration near the wall increase (erf, without wall), but they are equivalent among each other. 


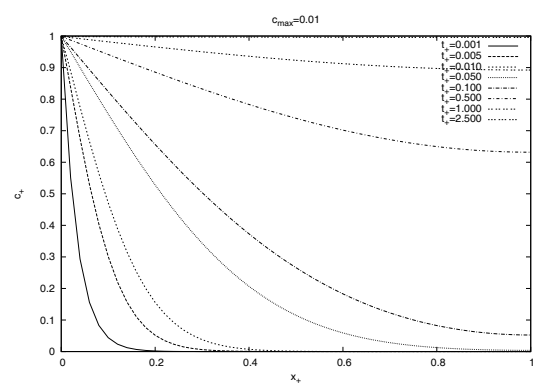

Figure 4: Time-dependent change of the spatial distribution of the local saturation level between $t_{+}=0,001$ and $t_{+}=2,5$ with $c_{\max }^{G}=0.01$.

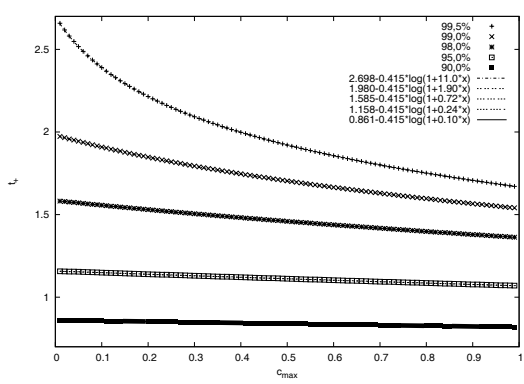

Figure 5: Isolines of the mean saturation level in a restricted domain over $c_{\max }^{G} \times t_{+}$and its modelling result.

The unsteady derive of the relative saturation level $c_{+}=c^{G} / c_{\max }^{G}$ is defined with following equation:

$$
\frac{\partial}{\partial t_{+}}\left(\rho_{+} c_{+}^{G}\right)=\frac{\partial}{\partial x_{+j}}\left(\frac{\rho_{+}}{1-c_{\max }^{G} c_{+}^{G}} \frac{\partial c_{+}^{G}}{\partial x_{+_{j}}}\right)
$$

As shown this time-dependent change of saturation level depends on the saturation concentration $c_{\max }^{G}$.

To stop an unsteady simulation with $c_{\max }^{G}=0,01$ the void time-scale $t_{+}=2,5$ seems to be great enough, because the mean saturation level has reached the maximum and the diffusion process is nearly completed. Analysing the isolines of the mean saturation level (Fig. 5), it is shown that the wanted time scale $t_{+}$has to be much higher to define the saturation process as completed (mean saturation level $>99 \%$ ) for low saturation concentrations $c_{\max }^{G}$.

The estimation of a time $T$, for what a domain with the length $L$ is approximately saturated, has to be between $t_{+}=2,0$ and $t_{+}=2,7$. A propose for an over-all approximation would be $t_{+}=2,5$ or:

$$
\frac{T D}{L^{2}}=t_{+} \approx 2,5 \quad \Rightarrow \quad T \approx \frac{5 L^{2}}{2 D}
$$

A finer approximation considering the saturation concentration $c_{\max }^{G}$ would be:

$$
T=\left[2,7-0.415 \ln \left(1+11 c_{\max }^{G}\right)\right] \frac{L^{2}}{D}<2,7 \frac{L^{2}}{D}
$$

For this model more than $99.5 \%$ of the maximum suspendible gas mass would be absorbed in the carrier phase. 


\section{Conclusion}

Modelling the diffusion process of a suspension in a liquid carrier phase by eq. (23) is possible with two kinds of boundary conditions:

1. Given concentration value at the phase interface $\left.c\right|_{x=0}=c_{\max }$

2. Modelled mass flow at the interface by eq. (21)

The simulation results of both boundary condition types are equivalent. If the diffusion process is executed far away from a domain restricting wall the simulation results are equivalent the exact solution

$$
c^{G}=\left[1+\frac{1-c_{\max }^{G}}{c_{\max }^{G}\left(1-\operatorname{erf}\left(\frac{x}{2 \sqrt{D t}}\right)\right)}\right]^{-1}
$$

of a heat equation (eq. (24)) additionally.

The characteristic time of an approximately completed diffusion process in a restricted domain, after what the mean local saturation level has nearly reached the maximum ( $>99.5 \%$ ) is restricted by $T<2,7 \frac{L^{2}}{D}$ and is given by eq. (32) depending on the domain length $L$, the diffusion parameter $D$ and the saturation concentration $c_{\max }^{G}$.

\section{References}

[1] R. V. Calabrese \& S. Middleman, The Dispersion of Discrete Particles in a Turbulent Fluid Field; AIChE Journal, Vol. 25(6), S. 1025-1035, 1979.

[2] R. Clift, J. R. Grace \& M. E. Weber, Bubbles, Drops and Particles; Academic Press, New York, 1978

[3] C. T. Crowe, M. Sommerfeld \& Y. Tsuji , Multiphase Flows with Droplets and Particles; CRC Press LLC, 1998.

[4] G. T. Csanady, Turbulent Diffusion of Heavy Particles in the Atmosphere; J. Atm. Sc., Vol.20, S.201-208, 1963.

[5] D. A. Drew , Mathematical Modelling of Two-Phase Flow; Ann. rev. Fluid Mech., Vol. 15, S. 261-291, Ann. Rev. Inc, 1983.

[6] H. Grad, On the Kinetic Theory on Rarefied Gases; Communications on Pure and Applied Mathematics, Vol.2, Nr.4, S. 331-407, 1949.

[7] R. I. Issa \& P. J. Oliveira , Accounting for Non-Equilibrium Turbulent Fluctuations in the Eulerian Two-Fluid Model by Means of the Notion of Introduction Period; The Third International Conference on Multiphase Flow ICMF'98, Lyon 1998.

[8] J. T. Jenkins \& M. W. Richman, Grad's 13-Moment-System for a Dense Gas of Inelastic Spheres; Arch. Ration. Mech. Anal., Vol.87, S. 355-177, 1985.

[9] P. K. Khosla \& S. G. Rubin, A Diagonally Dominant Second-Order Accurate Implicit Scheme; Computers Fluids, Vol. 2, S. 207-209, 1974.

[10] M. R. Maxey \& J. J. Riley, Equation of Motion for a Small Rigid Sphere in a Nonuniform Flow; Physics of Fluids, Vol. 26, S. 883-889, 1983. 
[11] R. A. Milikan, The General Law of Fall of a Small Particle through a Gas, and its Bearing upon the Nature of Moleculare Reflection from Surfaces; Phys. Rev., Vol. 22, 1923.

[12] F. Odar \& W. S. Hamilton, Forces on a Sphere accelerating in a viscous fluid, Vol. 18, S. 302 ff., 1964.

[13] P. J. Oliveira, Computer Modeling of Multidimensional Multiphase Flow and Application to T-Junctions; PhD Thesis, Empirial College London, 1992.

[14] W. A. Sirignano , Fluid Dynamics and Transport of Droplets and Sprays; Cambridge University Press, 1999.

[15] S. L. Soo , Fluid Dynamics of Multiphase Systems; University of Illinois, Blaisdell Publishing Company, 1967.

[16] C.-M. Tchen, Mean Value and Correlation Problems Connected with the Motion of Small Particles Suspended in a Turbulent Fluid; Martinus Nijhoff, Den Haag 1947.

[17] C. Vit, I. Flour \& O. Simonin , Modelling of Confined Bluff Body Flow Laden with Polydispersed solid particles; Two-Phase Flow Modelling and Experimentation 1999, Edizioni ETS Pisa, 1999

[18] L.-P. Wang \& S. E. Stock , Dispersion of Heavy Particles by Turbulent Motion; J. Atmos. Science, Vol.50, S. 1897-1913, 1993.

[19] L. P. Yarin \& G. Hetsroni , Turbulence Intensity in Dilute Two-Phase Flows 1-3; Int. J. Multiphase Flow, Vol. 20., No. 1, S.1-44, Elsevier Science Ltd., 1994.

[20] D. Z. Zhang \& A. Prosperetti , Averaged Equations for Inviscid Disperse Two-Phase Flow; J. Fluid Mech., Vol. 267, S. 185-219, Cambridge University Press, 1994. 